\title{
An Even Data-Distribution Protocolfor Highly Dynamic VANET
}

\author{
I.Bildass Santhosham ${ }^{1}$, I.Rinisha prem priya ${ }^{2}$, S. kiruba preethi ${ }^{3}$ \\ Assistant professor, ECE, CSICE, The Nilgiris, India ${ }^{l}$ \\ UG student, ECE, CSICE, The Nilgiris, India ${ }^{2}$ \\ UG student, ECE, CSICE, The Nilgiris, India ${ }^{3}$
}

\begin{abstract}
Vehicular ad -hoc network (VANET) has a problem called high mobility and uneven distribution of vehicles which affect the performance of routing. The high mobility may changes arrangements of a network, and the uneven distribution of vehicles leads to node failures due to network partition; In an urban environment the high density of vehicle cause drastic wireless contentions. In this paper, we use the Even Data Distribution (EDD) protocol to make uneven distribution of data transmission in the vehicular ad-hoc network to even distribution. In which the high mobility of vehicles in urban areas causes loss of data due to uneven distribution in order to reduce the impact of uneven distribution we transform it into even distribution using EDD protocol.
\end{abstract}

Keywords:EDD, Uneven distribution, Vehicular ad-hoc network, Even distribution.

\section{Introduction}

Vehicular Ad Hoc Network (VANET) is a spontaneous creation of a wireless network for data exchange. It is a sub form of Mobile Ad Hoc Network (MANET) that uses a moving car as nodes to create mobile network that establishes communication among nearby vehicle and roadside base station with an aim of providing efficient and safe transportation and aims to compose vehicular self -organizing network for intelligent transportation system (ITSs). Each vehicle equipped with communication device can act not only as a communication node, but also as a wireless router. Many distributed applications for vehicle have been proposed for supporting traffic safety, commercial demand, military uses, and other requirements of communication in VANETs.

Many issues arise towards running vehicular ad hoc networks to provide an improvement to driver behavior, with the aim of reducing automobile accidents. Thus, Itspecifies the main important challenges from the technical perspectives such as signal fading, bandwidth limitations, connectivity, small Effective diameter, security and privacy, routing. VANETs are designed to provide communication of vehicles. In VANETs two types of communications are possible, i.e. vehicle-to-vehicle (V2V) and roadside to vehicle communication (R2V) communication. Vehicular Ad hoc Networks (VANETs) is an infrastructure less network. For each vehicle's wireless network there is acoverage range that exists from 100 to 300 meters, so end-to-end communication in a larger distance is possible. In VANET to transmit a message (data) from a source node to destination node requires data should pass through several intermediate nodes. Vehicles also communicate with Road Site Unit (RSU) that increases communication range.Even Data Distribution is a Proactive (Table Driven) Protocol, In which Every node will maintain routing information for all other destinations. Routing information must be updated periodically. An EDD updated message consists of three fields.

- Destination address: It defines the destination end point, which receive information from any given sender. The original packet should include source and destination address.

- Sequence number: Protocol specifies that frames need to be numbered. This is done by using a sequence number. One important consideration is the range of the sequence number. In order to minimize the frame size range of sequence number should be small. If the field is $\mathrm{m}$ bits long, the sequence numbers start from 0 to $2^{\mathrm{m}}-1$ and then repeated.

- Hop count: The hop count is defined as number of nodes the packet is forwarded to reach destination. The cost of passing through a network is the same, it is one hop count. For example, if a packet passes through 5 nodes to reach the destination, the total count is 5 hop counts.

\section{Related work}

The most popular packet routing protocols in networks are categorized as link state and distance vector.

.Link State Routing Protocol : The link state protocol is performed by switching node in the network.It is build by shortest path .The basic concept is that every node constructs a map of the network, in the form of a graph,this shows which nodes are connected to which other nodes.Each nodes are independently calculates the next best path from it to every possible destination.Each collection of best paths will then form each node's routing table. 
Distance Vector Routing Protocol: Distance vector routing algorithm can cause both temporary and endurance loops due to updating the routing table with hard information. The looping problem can be eliminated, which requires the routers to correlate themselves mutually by secured messages in a stable environment. However, ad hoc networks are rapidly changing mobile environments. This is difficult to be used in the ad hoc networks.

The well-known implementation of distance vector routing, routing information protocol(RIP), has been successfully used within local networks. However, it has problem due to network topology changes. Unfortunately, they solve some problems and introduce others at the same time. The usefulness of the RIP to ad hoc environments is restricted due to the rapid topology change.

Both link state and distance vector routing algorithms are not suitable for ad hoc network routing.EDD is one of the proposed routing protocols for ad hoc networks. It aims to overcome the looping problem of RIP and also reduce uneven distribution.

\section{Routing strategy}

In EDD, each mobile node of an ad hoc network maintains a routing table, which lists all available destinations, the hop and next hop to each destination and a sequence number create by the destination node. Using such routing table stored in each mobile node, the packets are transmitted between the nodes .Each node of the ad hoc network updates the routing table periodically. The update packet starts out with a hop of one to direct connected nodes. This indicates each receiving neighbor is one hop away from the node.

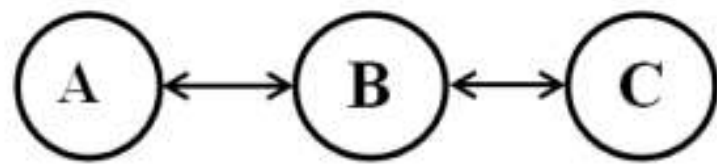

Fig1: Routing path

\begin{tabular}{|l|l|l|l|}
\hline destination & Next hop & Number of hops & Sequence number \\
\hline A & A & 0 & A46 \\
\hline B & B & 1 & B36 \\
\hline C & B & 2 & C28 \\
\hline
\end{tabular}

Table 1: Routing table

\section{System Model}

To address multi-hop routing for VANET's in an urban scenario, we make assumptions that each vehicle has a GPS device and a navigation system. In which the multi-hop routing means alternative routes for source and destination

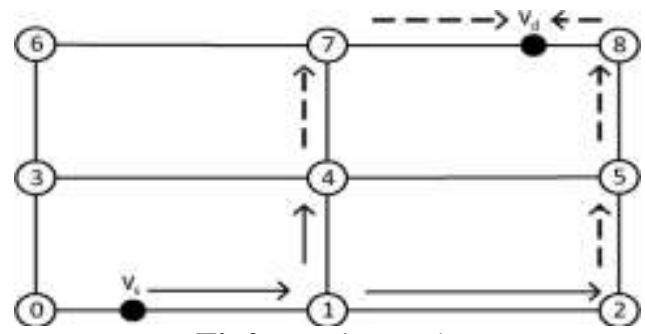

Fig2:Routing path

In this graph there are two vertices for routing selection, one is source vehicle Vs and other one is destination vehicle $\mathrm{V}_{\mathrm{d}}$. The routing path is done by three modules, depending on the packet delivery as follows.

- Dynamic route selection: It represents the changeable information of vehicles in the street, which actually determines the routing performance for a packet.It uses the concept of multi-hop routing in which it used to select an alternative path for data transmission.

- Next route selection:If the packet is at one End side, the identification of other end side is recorded in the header of the packet to avoid a routing loop problem. It is a serious network problem which happens when a data packet is continually routed through the same routers.

- Packet forwarding: It is relaying of packets from one node to another by nodes in a network. The original data of packet are transmitted from one node to another.

Performance evaluation:

We implement our proposed EDD in NS-2, it is a network simulator which runs on Linux.NS-2 provides routing and multicast protocols over wired and wireless networks. It works with two languages one is TCL (tool command language) other one is $\mathrm{c}++$. TCL is used for node creation and c++ used in the protocols. both are object oriented programming languages. 


\begin{tabular}{|l|l|}
\hline Simulation Parameler & Value \\
\hline Simulator & $\mathrm{NS}-2(\mathrm{v} 2.35)$ \\
\hline Simulation Area & $2000 \mathrm{~m} \times 1.500 \mathrm{~m}$ \\
\hline Total Number of Nodes & $1(10,150, \ldots .300$ \\
\hline Transmission Range & $250 \mathrm{~m}$ \\
\hline Channel Date Rate & $2 \mathrm{Mbps}$ \\
\hline Maximal Queuc I ength & 50 \\
\hline Number of CBR Connections & $20.40, \ldots .100)$ \\
\hline CBR Data Generating Rate & $2,4 . \ldots, 10$ \\
\hline Packet Size & $512 \mathrm{bytes}$ \\
\hline Min. Specd $\backslash$ Max. Specd & $30 \mathrm{~km} / \mathrm{h} \backslash 50 \mathrm{~km} / \mathrm{h}$ \\
\hline Simulation Time & $300 \mathrm{~s}$ \\
\hline Beacoul Interval & $1 \mathrm{~s}$ \\
\hline Inner Relaying Strategy & Deterministic Selection \\
\hline Radio Propagation Model & Nakagami-m as Fq. (2) \\
\hline Mac Layer & DCF of IEEE 802.11 \\
\hline Threshold Valuc $\approx$ & 0.01 \\
\hline
\end{tabular}

Table 2: Simulation table

\section{Output:}

Ns2 output has 3 files they are nam (.nam) ,trace (.tr) and Xgraph (.xgr)

- NAM:

Nam is TCL based animation tool for viewing network .

- TRACE:

The file written by an application to store coverage information or overall network information. Trace file is used to record the simulation events

- Xgraph:

It is a graphic representation of simulation results.

\section{XGRAPH}

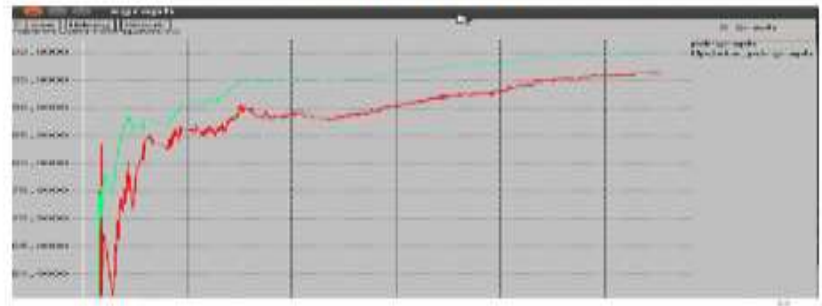

Routing in NS-2:(nam output)

Dynamic Route Selection

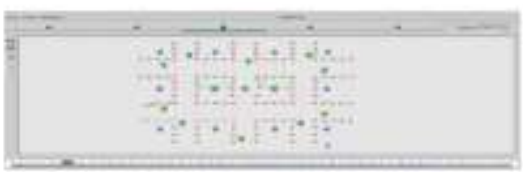

Next Route Selection

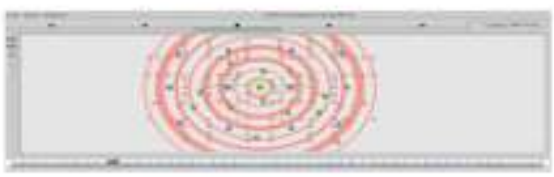

Packet Forwarding

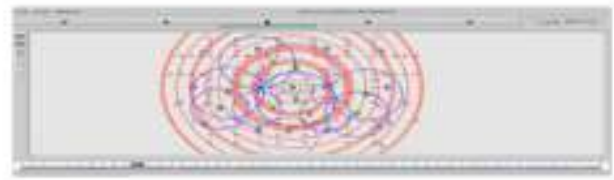

\section{Conclusion}

In this paper, we proposed a protocol called an Even Data Distribution protocol. Which make uneven distribution of data transmission in the vehicular ad-hoc network to an even distribution. In which the high mobility of vehicles in urban areas causes loss of data due to uneven distribution in order to reduce the impact of uneven distribution we transform it into even distribution using EDD protocol. In which it also increases the packet delivery ratio. The packet delivery ratiodepends on packet received by the destination and the packet generated by source. 


\section{Acknowledgement}

We would like to thank, Assistant Professor Bildass Santhosam for his immense support and guidance. He really gave us many helpful suggestions in tracing and modelling of VANET.

\section{Reference}

[1]. O. Andrisano, R. Verdone, and M. Nakagawa, "Intelligent transportation systems: The role of third generation mobile radio networks," IEEE Commun. Mag., vol. 38, no. 9, pp. 144-151, Sep. 2000

[2]. F. Li and Y. Wang, "Routing in vehicular ad hoc networks: A survey," IEEE Veh. Technol. Mag., vol. 2, no. 2, pp. 12-22, Jun. 2007

[3]. V. Kumar, S. Mishra, and N. Chand, “Applications of VANETs: Present \& future," Commun. Netw., vol. 5, no. 1B, pp. 12-15, 2013.

[4]. J. Nzouonta, N. Rajgure, G. Wang, and C. Borcea, "VANET routing on city roads using real-time vehicular traffic information," IEEETrans. Veh. Technol., vol. 58, no. 7, pp. 3609-3626, Sep. 2009.

[5]. Broch Joch, Maltz David A., etc.: A performance Comparison of Multi-Hop Wireless Ad Hoc Network Routing Protocols, Fouth AnnualACM/IEEE International Conference on Mobile Computing and Networking, 1998. 